\title{
A genetically trained simplified ANFIS controller to control nonlinear MIMO systems
}

\begin{abstract}
This paper presents a simplified ANFIS (Adaptive Neuro-Fuzzy Inference System) structure acting as a PID-like feedback controller to control nonlinear multi-input multi-output (MIMO) systems. Only few rules have been utilized in the rule base of this controller to provide the control actions, instead of the full combination of all possible rules. As a result, the proposed controller has several advantages over the conventional ANFIS structure particularly the reduction in execution time without sacrificing the controller performance, and hence, it is more suitable for real time control. In addition, the real-coded genetic algorithm (GA) has been utilized to train this MIMO ANFIS controller, instead of the hybrid learning methods that are widely used in the literature. Consequently, the necessity for the teaching signal required by other techniques has been eliminated. Moreover, the GA was used to find the optimal settings for the input and output scaling factors for this controller, instead of the widely used trial and error method. To demonstrate the accuracy and the generalization ability of the proposed controller, two nonlinear MIMO systems have been selected to be controlled by this controller. In addition, this controller robustness to output disturbances has been also evaluated and the results clearly showed the remarkable performance of this MIMO controller.
\end{abstract}

Keyword: ANFIS; Genetic algorithms; Neuro-fuzzy systems; Nonlinear MIMO systems 